\title{
EDUARDO TORROJA
}
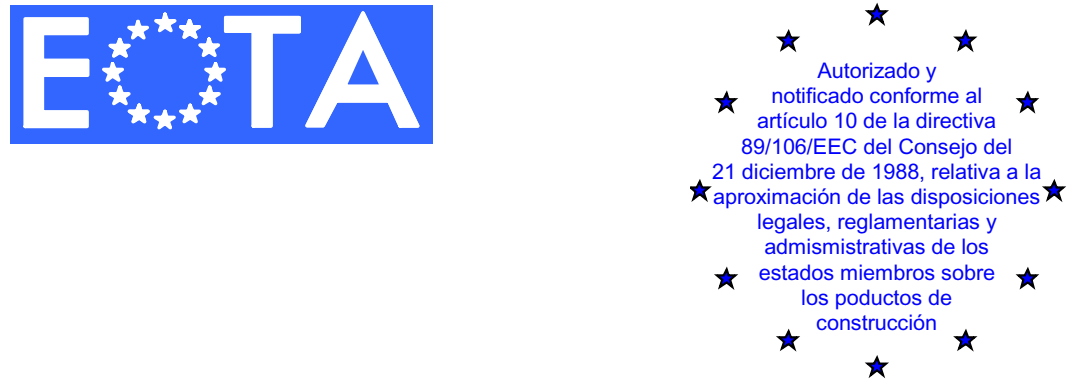
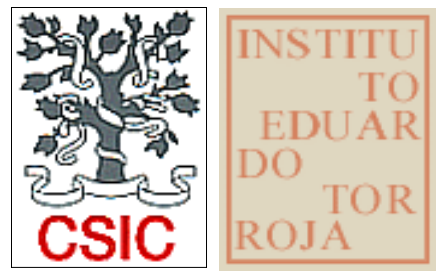

MIEMBRO DE EOTA EOTA MEMBER

\section{RELACIÓN DE DOCUMENTOS DE IDONEIDAD TÉCNICA EUROPEO (DITE) CONCEDIDOS Y ACTUALIZADOS EN EL AÑO 2010}

\begin{abstract}
DITE 04 - 0100
ANCLAJE GRANDES CARGAS INDEX SLVT

INDEX - Técnicas Expansivas S.L. c/ Segador s/n; Polígono industrial La Portalada II; 26006 LOGROÑO. España Sistema anclajes de expansión Los anclajes INDEX tipo SLVT de métricas M8, M10, M12, M16, M20 y M24 son anclajes fabricados en acero bicromatado, previstos para insertarse en un taladro y fijarse por medio de una expansión controlada por par de apriete. Los anclajes deben usarse para situaciones expuestas a cargas estáticas o cuasi-estáticas en hormigón armado o en masa, de peso normal (no aligerado).

Los anclajes exclusivamente pueden fijarse en hormigón no fisurado. Sólo pueden usarse en ambientes interiores secos.

Las previsiones indicadas en este DITE están basadas en una vida útil, estimada de 50 años, para el anclaje.

VALIDEZ: 12/11/2014

(actualizado 24/05/2010).
\end{abstract}

\section{DITE 05-0242}

\section{ANCLAJE INDEX MTH, MTH-A4}

INDEX - Técnicas Expansivas S.L.

C/ Segador s/n; Polígono industrial La Portalada II; 26006 LOGROÑO. España. Sistema anclajes de expansión

Los anclajes INDEX tipo MTH de métricas M6, a M20 son anclajes fabricados en acero galvanizado. Los anclajes INDEX tipo MTH-AH de métricas M6 a M20 son anclajes fabricados en acero inoxidable. Ambos están previstos para insertarse en un taladro y fijarse por medio de una expansión controlada por par de apriete. Los anclajes deben usarse para situaciones expuestas a cargas estáticas o cuasi-estáticas en hormigón armado o en masa, de peso normal (no aligerado).
Los anclajes exclusivamente pueden fijarse en hormigón no fisurado. Los anclajes INDEX MTH sólo pueden usarse en ambientes interiores secos. Los anclajes INDEX MTH-A4 pueden usarse en ambientes exteriores secos y también en los expuestos a distintas condiciones atmosféricas externas (incluyendo ambientes industriales y marinos).

Las previsiones indicadas en este DITE están basadas en una vida útil, estimada de 50 años, para el anclaje.

VALIDEZ: 08/11/2015

(actualizado 08/11/2010).

\section{DITE 06-0058}

\section{POLYDAN PLUS FM}

DERIVADOS ASFÁLTICOS

NORMALIZADOS, S.A. (DANOSA)

Avda. Somosierra $n^{\circ} 8 ; 28700$

SAN SEBASTIÁN DE LOS REYES

(Madrid)-España

Sistemas monocapa de impermeabilización de cubiertas

POLYDAN PLUS FM es un sistema de impermeabilización de cubiertas de una sola capa, de láminas flexibles de betún modificado con SBS (EstirenoButadieno-Estireno) fijadas mecánicamente, cobre cubiertas, con pendientes mayores del $1 \%$.

VALIDEZ: 01/02/2011

(actualizado 23/01/2010).

\section{DITE 06-0089}

\section{SISTEMA COTETERM}

PAREX MORTEROS SAU

c/ Italia 13-21. Polígono Industrial Pla de Llerona.08520 Las Franquesas del Vallés. Barcelona.

El Sistema COTETERM está previsto para uso como aislamiento térmico por el exterior de muros de edificación. Los muros pueden ser de albañilería (ladriIlo, bloque, o bien de hormigón, hecho in situ o a base de paneles prefabricados). El Sistema está destinado a dotar al paramento sobre el que se instale de un aislamiento térmico satisfactorio y se une al soporte mediante adhesivo y fijaciones mecánicas suplementarias. El Sistema se compone de elementos no portantes y no participa en la estabilidad ni en la estanquidad al aire del soporte sobre el que se aplica, pero en cambio contribuye a su durabilidad al protegerle frente a los agentes naturales.

VALIDEZ: 01/09/2011

(actualizado 02/08/2010)

\section{DITE 07-0054}

SISTEMA TRADITERM

GRUPO PUMA SL

c/ Conrado del Campo no 2, $1^{\text {a }}$ planta.

Parque industrial Trévenez.

29590 Campanillas (Málaga).

El Sistema TRADITERM está previsto para uso como aislamiento térmico por el exterior de muros de edificación. Los muros pueden ser de albañilería (ladrillo, bloque, o bien de hormigón, hecho in situ o a base de paneles prefabricados). El Sistema está destinado a dotar al paramento sobre el que se instale de un aislamiento térmico satisfactorio y se une al soporte mediante adhesivo y fijaciones mecánicas suplementarias. El Sistema se compone de elementos no portantes y no participa en la estabilidad ni en la estanquidad al aire del soporte sobre el que se aplica, pero en cambio contribuye a su durabilidad al protegerle frente a los agentes naturales.

VALIDEZ: 09/03/2012

(actualizado 16/08/2010). 


\section{DITE 07-0223}

\section{F-0101}

FAPLISA (Fabricación y Aplicación de Pinturas Especiales S.A.)

c/ Paloma $\mathrm{n}^{\circ}$ 13; Polígono Industrial

Los Gallegos. 28946 FUENLABRADA

(Madrid) España.

Producto de señalización vial horizontal

F-0101 es una pintura de señalización vial horizontal (tal como se define en EN 1871) que se emplea como material base para aplicarlo sobre la calzada con o sin postmezclado de agregados antideslizantes y/o microesferas de vidrio, con objeto de construir una marca vial. La pintura se comercializa con las correspondientes instrucciones de aplicación que incluyen los tipos y proporciones de agregados antideslizantes y/o microesferas de vidrio de postmezclado.

VALIDEZ: 12/12/2012

(actualizado 30/12/2010).

\section{DITE 07-0224}

\section{F-2005}

FAPLISA (Fabricación y Aplicación de

Pinturas Especiales S.A.)

c/ Paloma $n^{\circ} 13$; Polígono Industrial

Los Gallegos. 28946 FUENLABRADA

(Madrid) España.

Producto de señalización vial horizontal F-2005 es un termoplástico de señalización vial horizontal (tal como se define en EN 1871) que se emplea como material base para aplicarlo sobre la calzada con o sin postmezclado de agregados antideslizantes y/o microesferas de vidrio, con objeto de construir una marca vial. La pintura se comercializa con las correspondientes instrucciones de aplicación que incluyen los tipos y proporciones de agregados antideslizantes y/o microesferas de vidrio de postmezclado.

VALIDEZ: 12/12/2012

(actualizado 30/12/2010).

\section{DITE 07-0225}

\section{F-1005S1}

FAPLISA (Fabricación y Aplicación de Pinturas Especiales S.A.)

c/ Paloma $n^{\circ} 13$; Polígono Industrial Los Gallegos. 28946 FUENLABRADA (Madrid) España

Producto de señalización vial horizontal F-1005S1 es una pintura de señalización vial horizontal (tal como se define en EN 1871) que se emplea como material base para aplicarlo sobre la calzada con o sin postmezclado de agregados antideslizantes y/o microesferas de vidrio, con objeto de construir una marca vial. La pintura se comercializa con las correspondientes instrucciones de aplicación que incluyen los tipos y proporciones de agregados antideslizantes y/o microesferas de vidrio de postmezclado.

VALIDEZ: 12/12/2012

(actualizado 30/12/2010).

\section{DITE 07-0227}

\section{F-2009}

FAPLISA (Fabricación y Aplicación de

Pinturas Especiales S.A.)

c/ Paloma $n^{\circ} 13$; Polígono Industrial

Los Gallegos. 28946 FUENLABRADA

(Madrid). España.

Producto de señalización vial horizontal F-2009 es un termoplástico de señalización vial horizontal (tal como se define en EN 1871) que se emplea como material base para aplicarlo sobre la calzada con o sin postmezclado de agregados antideslizantes y/o microesferas de vidrio, con objeto de construir una marca vial. La pintura se comercializa con las correspondientes instrucciones de aplicación que incluyen los tipos y proporciones de agregados antideslizantes y/o microesferas de vidrio de postmezclado.

VALIDEZ: $12 / 12 / 2012$

(actualizado 30/12/2010).

\section{DITE 08-0169 \\ APPROVED THROUGHBOLT AWA, AWA SS A-4}

JCP OWLETT-JATON

Unit 07 Princess Court; Horace Road; KingstonSurrey. KT1 2SL;

UNITED KINGDOM (REINO UNIDO) Sistemas de anclajes de expansión Los anclajes APPROVED THROUGHBOLT tipo AWA de métricas M6, M8, M10, M12, M14, M16, y M20 son anclajes de par controlado fabricados en acero galvanizado. Los anclajes APPROVED THROUHBOLT tipo AWA SS A-4 de métricas M6 a M20 son anclajes de par controlado fabricados en acero inoxidable. Ambos están previstos para insertarse en un taladro y fijarse por medio de una expansión controlada por par de apriete.

VALIDEZ: 08/11/2015

(actualizado 8/11/2010).

\section{DITE 08-0388}

\section{ANCHOR WÜRTH W-F, W-F A4}

WÜRTH España S.A.

c/ Joiers no 21; Polígono Industrial Riera de Caldes; 08140 PALAU SOLITÀ I PLEGAMANS (Barcelona) España Sistemas de anclajes de expansión Los anclajes WÜRTH W-F de métricas M6 a M20 son anclajes de par controlado fabricados en acero galvanizado. Los anclajes WÜRTH W-F A4 de métricas M6 a M20 son anclajes de par controlado fabricados en acero inoxidable. Ambos están previstos para insertarse en un taladro y fijarse por medio de una expansión controlada por par de apriete. VALIDEZ: 08/11/2015.

\section{DITE 09-0154 \\ CALIPLAC}

CUBIERTAS ALIGERADAS

TERMOACÚSTICAS S.L.

c/ Río Cúa, 27. Polígono Industrial de El Bierzo. 24560 TORAL DE LOS VADOS (León). España

Sistema para cerramiento de cubiertas con paneles sándwich ligeros

El Sistema CALIPLAC $₫$ está previsto para uso como cierre autoportante aislante de cubiertas.

Los paneles CALIPLAC $®$ son adecuados para uso en ambiente interior en condiciones de humedad normales.

El Sistema no tiene funciones estructurales ni tiene influencia significativa en la estabilidad del edificio.

VALIDEZ: 28/05/2015.

\section{DITE 10-0008 \\ SISTEMA DE POSTESADO MONOTORON STRONGHOLD}

CTT- STRONGHOLD S.A.

Passeig de Gracia n ${ }^{\circ} 11$, esc. B. $1^{\text {a }}$ Planta. BARCELONA (España)

Sistema de pretensazo de estructuras Kit de postesado (tendón) adherente y no adherente para estructuras (principalmente forjados de edificación y tableros de puentes) compuesto básicamente por un cordón de postesado, anclajes, vaina plástica y sus acopladores y otros elementos en la zona de anclaje tales como refuerzo y te de inyección. Los tendones/kit de postesado tienen los siguientes usos:

- Tendones para postesado interior adherente en estructuras de hormigón o estructuras mixtas.

- Tendones para postesado interior no adherente en estructuras de hormigón o estructuras mixtas.

Así mismo, puede utilizarse para las siguientes categorías de uso opcionales:

- Tendones para postesado exterior o interior (adherente o no adherente) para refuerzo estructural o reparación.

VALIDEZ: 26/06/2015.

\section{DITE 10-0022}

\section{SISTEMA DEPROT ${ }^{\circledR}$}

PANELES PÉTREOS S.L.

Camino de Las Ánimas no 8. 09200 MIRANDA DE EBRO (Burgos). España Kit de elementos prefabricados para aislamiento térmico exterior en muros (Vêtures)

El Sistema DEPROT® está previsto para uso como aislamiento térmico por el 
exterior de muros de edificación. Los muros pueden ser de albañilería (ladriIlo, bloque) o bien de hormigón (hecho in situ o a base de paneles prefabricados). El kit está destinado a dotar al paramento sobre el que se instale de un aislamiento térmico satisfactorio. El kit se compone de elementos no portantes y no participa en la estabilidad ni en la estanquidad al aire del soporte sobre el que se aplica, pero en cambio contribuye a su durabilidad al protegerle frente a la intemperie.

VALIDEZ: 05/05/2015.

\section{DITE 10-0050 \\ LUSO SPRAY TERMO-SPRAY. BLAN- CO Cod. TPVS-101}

VOUGACOR - Productos Sinalizaçao Rodoviária, Lda

Zona Industrial de Mamodeiro. Lote 14. Mamodeiro. 3810-783 AVEIRO. Portugal Producto de señalización vial horizontal LUSO SPRAY TERMO-SPRAY BLANCO Cod. TPVS-101 es un termoplástico de señalización vial horizontal (tal como se define en EN 1871) que se emplea como material base para aplicarlo sobre la calzada con o sin postmezclado de agregados antideslizantes y/o microesferas de vidrio, con objeto de construir una marca vial. La pintura se comercializa con las correspondientes instrucciones de aplicación que incluyen los tipos y proporciones de agregados antideslizantes y/o microesferas de vidrio postmezclado.

VALIDEZ: 09/03/2015.

\section{DITE 10-0054}

\section{DANOPOL HS FM}

DERIVADOS ASFÁLTICOS NORMALIZADOS S.A. (DANOSA)

Sector 9, Polígono Industrial. 19290

FONTANAR (Guadalajara). España

Sistemas monocapa de impermeabilización de cubiertas

DANOPOL HS FM es un sistema de impermeabilización de cubiertas de una sola capa, de láminas flexibles sintéticas de PVC reforzadas, fijadas mecánicamente sobre cubiertas con pendientes mayores del $1 \%$.

VALIDEZ: 23/02/2015.

\section{DITE 10-0096}

\section{REVETON CUBIERTAS}

MATERIS PAINTS ESPAÑA S.L.

C/ Francia, 7-Polígono Industrial Pla de Llerona. 08520 LES FRANQUESES DEL VALLÈS (Barcelona). España

Sistema de impermeabilización de cubiertas aplicado en forma líquida El Sistema de impermeabilización de cubiertas aplicado en forma líquida, ba- sado en polímeros en dispersión acuosa REVETON CUBIERTAS está constituido por resinas acrílicas, elastomérico, armado de aplicación "in situ", el cual una vez polimerizado conforma un revestimiento elástico, en forma de una capa totalmente adherida al soporte (hormigón, mortero, cerámica).

El espesor mínimo del producto aplicado debe ser de $1,5 \mathrm{~mm}$ y el rendimiento mayor de 2,5 kg/m².

VALIDEZ: $17 / 04 / 2015$.

\section{DITE 10-0116 \\ AIR-BUR TERMIC}

BUR 2000 S.A.

C/ Progreso 18-20. 08850 GAVÀ

(Barcelona) España

Productos con componentes

reflectivos de calor

El sistema AIR-BUR TERMIC está constituido por una o dos láminas extremas reflectivas (de baja emisividad $<0,10$ ) de aluminio (min 98,5\% de pureza), unidas por termo-soldado a varias láminas de polietileno y de aluminio. El espesor total de las láminas varía entre 3,5 y 9 mm.

VALIDEZ: 11/05/2015.

\section{DITE 10-0121 \\ DESMOPOL}

TECNOPOL SISTEMAS S.L.

Polígono Ind. "Z". Calle de la Prensa $n^{\circ}$ 5. 08150 PARETS DEL VALLÈS (Barcelona). España

Sistema de impermeabilización de cubiertas aplicado en forma líquida

El Sistema de impermeabilización de cubiertas aplicado en forma líquida, basado en poliuretano DESMOPOL está constituido por resinas de poliuretano monocomponente, elastomérico no armado de aplicación "in situ", el cual una vez polimerizado conforma un revestimiento elástico, en forma de una capa toalmente adherida al soporte (hormigón, mortero, cerámica).

El espesor mínimo del producto aplicado debe ser de $1,2 \mathrm{~mm}$ y el rendimiento mayor de $1,5 \mathrm{~kg} / \mathrm{m}^{2}$.

El Sistema incluye una malla de geotextil como refuerzo en los puntos singulares de la cubierta y un revestimiento superficial DESMOPOL-ADY para un uso de carga P3 y vida útil de 10 años (consumo mínimo $150 \mathrm{~g} / \mathrm{m}^{2}$.

VALIDEZ: 12/03/2014.

\section{DITE 10-0142}

MORCEM ${ }^{\circledR}$ ISOLAMENTO

PEGACOL Lda.

Fornos de cima - Calhandriz. 2615-641 ALVERCA (Portugal)
Sistema de aislamiento térmico exterior El sistema MORCEM $®$ Isolamento está previsto para uso como aislamiento térmico por el exterior de muros de edificación. Los muros pueden ser de albañilería (ladrillo, bloque, o bien de hormigón, hecho in situ o a base de paneles prefabricados). El sistema está destinado a dotar al paramento sobre el que se instale de un aislamiento térmico satisfactorio.

VALIDEZ: 09/03/2012.

\section{DITE 10-0162 \\ HYPERTECTUM}

ETS EUROPE BVBA

Doggenhoustraat $n^{\circ}$ 3, B-2520 Ranst. AMBERES(Bélgica)

Sistema de impermeabilización de cubiertas aplicado en forma líquida

El Sistema de impermeabilización de cubiertas aplicado en forma líquida, basado en poliuretano HYPERTECTUM está constituido por resinas de poliuretano monocomponente, elastomérico no armado de aplicación "in situ", el cual una vez polimerizado conforma un revestimiento elástico, en forma de una capa totalmente adherida al soporte (hormigón, mortero, cerámica).

El espesor mínimo del producto aplicado debe ser de 1,2 $\mathrm{mm}$ y el rendimiento mayor de $1,5 \mathrm{~kg} / \mathrm{m}^{2}$.

El Sistema incluye una malla de geotextil como refuerzo en los puntos singulares de la cubierta y un revestimiento superficial HYPERTECTUM topcoat para un uso de carga P3 y vida útil de 10 años (consumo mínimo $150 \mathrm{~g} / \mathrm{m}^{2}$.

VALIDEZ: 02/12/2014.

\section{DITE 10-0226}

\section{SPRAY BLANCO CHR P7}

PRODUCTOS DÍEZ S.A.

C/ San Cesáreo nº 6. Z.I. Villaverde

Alto. 28021 MADRID. España

Producto de señalización vial horizontal Tipo II

73015 SPRAY BLANCO CHR P7, es un termoplástico de señalización vial horizontal (tal como se define en EN 1871) que se emplea como material base para aplicarlo sobre la calzada con o sin postmezclado de agregados antideslizantes y/o microesferas de vidrio, con objeto de construir una marca vial. La pintura se comercializa con las correspondientes instrucciones de aplicación que incluyen los tipos y proporciones de agregados antideslizantes $y / 0$ microesferas de vidrio postmezclado.

VALIDEZ: 18/08/2015. 


\section{DITE 10-0231}

TERMOK8 MINERALE L.V.

I.V.A.S. Industria Vernici S.p.A.

Via Bellaria, 40. 47030 SAN MAURO

PASCOLI (FC). Italia

Sistema de aislamiento térmico por el exterior con revoco

El sistema TERMOK8 MINERALE L.V. está previsto para uso como aislamiento térmico por el exterior de muros de edificación. Los muros pueden ser de albañilería (ladrillo, bloque, o bien de hormigón, hecho in situ o a base de paneles prefabricados). El Sistema está destinado a dotar al paramento sobre el que se instale, de un aislamiento térmico satisfactorio y se une al soporte mediante adhesivo y fijaciones mecánicas suplementarias. El Sistema se compone de elementos no portantes y no participa en la estabilidad ni en la estanquidad al aire del soporte sobre el que se aplica, pero en cambio contribuye a su durabilidad al protegerle frente a los agentes naturales.

VALIDEZ: 26/07/2015

(actualizado 23/02/2011).

\section{DITE 10 -0247}

VIALINE AC-3B; Cod. AC-121

MARCAS VIALES S.A.

Vega del Tajo s/n. Polígono Industrial

n 1 de Quer. 19209 QUER

(Guadalajara). España

Producto de señalización vial horizontal VIALINE AC-3B; Cod. AC-121 es una pintura de señalización vial horizontal (tal como se define en EN 1871) que se emplea como material base para aplicarlo sobre la calzada con o sin postmezclado de agregados antideslizantes y/o microesferas de vidrio, con objeto de construir una marca vial. La pintura se comercializa con las correspondientes instrucciones de aplicación que incluyen los tipos y proporciones de agregados antideslizantes $y / 0$ microesferas de vidrio de postmezclado.

VALIDEZ: 18/08/2015.

\section{DITE 10-0248}

\section{MARCALINE 1:1 Componentes A y B}

\section{Cod: DC-492}

MARCAS VIALES S.A.

Vega del Tajo s/n. Polígono Industrial no 1 de Quer. 19209 QUER

(Guadalajara). España

Producto de señalización vial horizontal MARCALINE 1:1 COMPONENTES A y B Code DC-492, plástico en frío de señalización vial horizontal (tal como se define en 1871) emplea como material base para aplicarlo sobre la calzada con o sin postmezclado de agregados antideslizantes y/o microesferas de vidrio, con objeto de construir una marca vial. La pintura se comercializa con las correspondientes instrucciones de aplicación que incluyen los tipos y proporciones de agregados antideslizantes y/o microesferas de vidrio de postmezclado. VALIDEZ: 18/08/2015.

\section{DITE 10-0296}

\section{IMPERMAX 2K}

KRYPTON CHEMICAL S.L.

$\mathrm{C} /$ Martí Franques $\mathrm{n}^{\circ} 12$. Polígono Ind. Les Tàpies. 43890 L'HOSPITALET DE

LLOBREGAT (Tarragona). España

Sistema de impermeabilización de cubiertas aplicado en forma líquida El producto de impermeabilización de cubiertas aplicado en forma líquida, basado en poliuretano "IMPERMAX $2 \mathrm{~K}^{\text {", }}$ está constituido por resinas de poliuretano bi-componente, elastomérico no armado de aplicación "in situ"; el cual una vez polimerizado conforma un revestimiento elástico, en forma de una capa totalmente adherida al soporte (hormigón, mortero, cerámica, aglomerado de madera tipo MDF y metal).

El espesor mínimo de IMPERMAX 2K aplicado debe ser de 1,9 $\mathrm{mm}$ y el rendimiento mayor de $2 \mathrm{~kg} / \mathrm{m}^{2}$.

VALIDEZ: 21/08/2015.

\section{DITE 10-0337}

\section{ATK 1000}

MERKA S.L.

C/ Diseminados, 16, Pab. 4-5-6 Telleriarte.

20230 LEGAZPI (Guipúzcoa). España

Sistema de impermeabilización de cubierta aplicado en forma líquida El producto de impermeabilización de cubiertas aplicado en forma líquida, basado en poliuretano "ATK 1000", está constituido por resinas de poliuretano bi-componente, elastomérico no armado de aplicación "in situ"; el cual una vez polimerizado conforma un revestimiento elástico, en forma de una capa totalmente adherida al soporte (hormigón, mortero, cerámica). Este Producto siempre debe ir protegido, esta protección evita el contacto de la membrana con la radiación UV.

El espesor mínimo del producto aplicado deber ser de $1,5 \mathrm{~mm}$ y el rendimiento mayor de $1,5 \mathrm{~kg} / \mathrm{m}^{2}$.

VALIDEZ: 17/04/2015.

\section{DITE 10-0340}

\section{CERESIT CEREPUR CS 58 2K}

TURK HENKEL KIMYA SAN TIC. A.S.

Kayisdagi Cad. Karaman Ciftligi Yolu Kar Plaza D Blok. Icerenkoy Estambul.

TURQUIA

Impermeabilización de cubiertas aplicado en forma líquida
El producto de impermeabilización de cubiertas aplicado en forma líquida, basado en poliuretano "CERESIT CEREPUR CS $582 \mathrm{~K}^{\prime}$, está constituido por resinas de poliuretano bi-componente, elastomérico no armado de aplicación "in situ"; el cual una vez polimerizado conforma un revestimiento elástico, en forma de una capa totalmente adherida al soporte (hormigón, mortero, cerámica). Este Producto siempre debe ir protegido, esta protección evita el contacto de la membrana con la radiación UV. El espesor mínimo del producto aplicado debe ser de 1,5 $\mathrm{mm}$ y el rendimiento mayor de $1,5 \mathrm{~kg} / \mathrm{m}^{2}$.

VALIDEZ: 17/04/2015.

\section{DITE 10-0341 \\ CERESIT CEREPUR}

TURK HENKEL KIMYA SAN TIC. A.S. Kayisdagi Cad. Karaman Ciftligi Yolu Kar Plaza D Blok. Icerenkoy Estambul.

TURQUIA

Impermeabilización de cubiertas aplicado en forma líquida

El producto de impermeabilización de cubiertas aplicado en forma líquida, basado en poliuretano "CERESIT CEREPUR", está constituido por resinas de poliuretano bi-componente, elastomérico no armado de aplicación "in situ"; el cual una vez polimerizado conforma un revestimiento elástico, en forma de una capa totalmente adherida al soporte (hormigón, mortero, cerámica).

El espesor mínimo del producto aplicado debe ser de 1,2 $\mathrm{mm}$ y el rendimiento mayor de $1,5 \mathrm{~kg} / \mathrm{m}^{2}$.

VALIDEZ: 02/12/2014.

\section{DITE 10-342 \\ ALP ELAST}

MATERIS PAINTS ESPAÑA S.L.

c/ Francia $n^{\circ} 7$. Polígono Industrial Plà de Llerona. 08520 LES FRANQUESES DEL VALLÈS (Barcelona). España.

Impermeabilización de cubiertas aplicado en forma líquida

El sistema de impermeabilización de cubiertas aplicado en forma líquida, basado en polímeros en dispersión acuosa ALP ELAST, está constituido por un copolímero estireno-acrílico monocomponente en dispersión acuosa, con cargas y pigmentos minerales y aditivos (antiespumantes, biocidas, etc.); el cual una vez polimerizado conforma un revestimiento elástico, en forma de una capa totalmente adherida al soporte (hormigón, mortero, cerámica). El sistema incluye una malla de fibra de vidrio (TEXNON 300) como refuerzo de la membrana. 
El espesor mínimo del producto aplicado debe ser de $1,5 \mathrm{~mm}$ y el consumo mayor de $2,5 \mathrm{~kg} / \mathrm{m}^{2}$.

VALIDEZ: 17/04/2015.

\section{DITE 10-343 \\ ELASTICO LP-70}

MATERIS PAINTS ESPAÑA S.L.

c/ Francia $n^{\circ} 7$. Polígono Industrial Plà de Llerona. 08520 LES FRANQUESES DEL VALLÈS (Barcelona). España. Impermeabilización de cubiertas aplicado en forma líquida

El sistema de impermeabilización de cubiertas aplicado en forma líquida, basado en polímeros en dispersión acuosa IMPERMEABILIZANTE ELASTICO LP70, está constituido por un copolímero estireno-acrílico mono-componente en dispersión acuosa, con cargas y pigmentos minerales y aditivos (antiespumantes, biocidas, etc.); el cual una vez polimerizado conforma un revestimiento elástico, en forma de una capa totalmente adherida al soporte (hormigón, mortero, cerámica). El sistema incluye una malla de fibra de vidrio (TEXNON 300) como refuerzo de la membrana.

El espesor mínimo del producto aplicado debe ser de $1,5 \mathrm{~mm}$ y el consumo mayor de $2,5 \mathrm{~kg} / \mathrm{m}^{2}$.

VALIDEZ: 17/04/2015.

\section{DITE 10-0344 \\ REVETON TEC PLUS}

MATERIS PAINTS ESPAÑA S.L.

c/ Francia $n^{\circ} 7$. Polígono Industrial Plà de Llerona. 08520 LES FRANQUESES DEL VALLÈS (Barcelona). España. Impermeabilización de cubiertas aplicado en forma líquida

El sistema de impermeabilización de cubiertas aplicado en forma líquida, basado en polímeros en dispersión acuosa REVETON TEC PLUS, está constituido por un copolímero estireno-acrílico mono- componente en dispersión acuosa, con cargas y pigmentos minerales y aditivos (antiespumantes, biocidas, etc.); el cual una vez polimerizado conforma un revestimiento elástico, en forma de una capa totalmente adherida al soporte (hormigón, mortero, cerámica). El sistema incluye una malla de fibra de vidrio (TEXNON 300) como refuerzo de la membrana.

El espesor mínimo del producto aplicado debe ser de $1,5 \mathrm{~mm}$ y el consumo mayor de $2,5 \mathrm{~kg} / \mathrm{m}^{2}$.

VALIDEZ: 17/04/2015.

DITE 10-0353

LASUR ${ }^{\circledR}$ TERM

APLICACIONES Y VENTAS SL (APLIVE SL)
Polígono Industrial El Gordillo.

Ctra. Sevilla-Brenes km 5,5. 41300-San José de la Rinconada. Sevilla. España.

Sistema de Aislamiento térmico por el exterior con revoco

El Sistema LASUR® TERM está previsto para uso como aislamiento térmico por el exterior de muros de edificación. Los muros pueden ser de albañileria (ladriIlo, bloque, o bien de hormigón, hecho in situ o a base de paneles prefabricados). El Sisema está destinado a dotar al paramento sobre el que se instale de un aislamiento térmico satisfactorio y se une al soporte mediante adhesivo y fijaciones mecánicas suplementarias. El Sistema se compone de elementos no portantes y no participa en la estabilidad ni en la estanquidad al aire del soporte sobre el que se aplica, pero en cambio contribuye a su durabilidad al protegerle frente a los agentes naturales.

VALIDEZ: 09/03/2012

(actualizado 16/08/2010).

\section{DITE 10-0380}

\section{SISTEMA RESI-ETANCH}

LPH S.A.

Lacropte B.P 32. 24380 VERGT

(Francia)

Sistema de impermeabilización de

cubiertas aplicado en forma líquida,

basado en poliuretanos

El Sistema de impermeabilización de cubiertas aplicado en forma líquida, basado en poliuretano RESI-ETANCH, está constituido por resinas de poliuretano monocomponente, elastomérico no armado de aplicación "in situ", el cual una vez polimerizado conforma un revestimiento elástico, en forma de una capa totalmente adherida al soporte (hormigón, mortero, cerámica).

El espesor mínimo del producto aplicado debe ser de $1,2 \mathrm{~mm}$ y el rendimiento mayor de $1,5 \mathrm{~kg} / \mathrm{m}^{2}$.

VALIDEZ: 02/12/2014.

\section{DITE 10-0381}

SISTEMA LABO MLE

LABO FRANCE S.A.

Z.I. de Ladoux - Rue Bleue. B.P.

70051. 63118 CEBAZAT (Francia)

Sistema de impermeabilización de cubiertas aplicado en forma líquida, basado en poliuretanos

El Sistema de impermeabilización de cubiertas aplicado en forma líquida, basado en poliuretano LABO-MLE, está constituido por resinas de poliuretano monocomponente, elastomérico no armado de aplicación "in situ", el cual una vez polimerizado conforma un revestimiento elástico, en forma de una capa totalmente adherida al soporte (hormigón, mortero, cerámica).

El espesor mínimo del producto aplicado debe ser de 1,2 $\mathrm{mm}$ y el rendimiento mayor de $1,5 \mathrm{~kg} / \mathrm{m}^{2}$.

VALIDEZ: 02/12/2014.

\section{DITE 10-0394 \\ SUPERFLEX}

GROUP D\&I

De Leescorfstraat 19. 2140 Amberes. Bélgica

Sistema de impermeabilización de cubierta aplicado en forma líquida, basado en poliuretanos

El Sistema de impermeabilización de cubiertas aplicado en forma líquida, basado en poliuretano SUPERFLEX, está constituido por resinas de poliuretano monocomponente, elastomérico, en forma de una capa totalmente adherida al soporte (hormigón, mortero, cerámica).

El espesor mínimo del producto aplicado debe ser de 1,2 $\mathrm{mm}$ y el rendimiento mayor de $1,5 \mathrm{~kg} / \mathrm{m}^{2}$.

VALIDEZ: 02/12/2014.

\section{DITE 10-0441}

TWINCRIL DOS COMPONENTES

MÁQUINA BLANCO - REF.: 6100 COMP-B/6001 COMP-A

RALVA S.A.

C/ Calahorra $n^{\circ}$ 2. 28864 AJALVIR

(Madrid). España

Producto de señalización vial horizontal TWINCRIL DOS COMPONENTES MÁQUINA BLANCO - REF.: 6100 COMPB/6001 COMP-A es una pintura de señalización vial horizontal (tal como se define en la Norma EN 1871) que se emplea como material base para aplicarlo sobre la calzada sin necesidad de materiales agregados. La pintura se comercializa con las correspondientes instrucciones de aplicación.

VALIDEZ: 30/12/2015.

\section{DITE 10-0442 \\ SEÑACRYL CARRETERAS BLANCO - REF.:3105}

RALVA S.A.

C/ Calahorra no 2. 28864 AJALVIR

(Madrid). España

Producto de señalización vial horizontal SEÑACRYL CARRETERAS BLANCO REF.:3105 es una pintura de señalización vial horizontal (tal como se define en la Norma EN 1871) que se emplea como material base para aplicarlo sobre la calzada sin necesidad de materiales agregados. La pintura se comercializa con las correspondientes instrucciones de aplicación.

VALIDEZ: 30/12/2015. 


\section{DITE 10-0443}

\section{CITICRYL D BLANCO - REF.:3129}

RALVA S.A

C/ Calahorra n 2. 28864 AJALVIR (Madrid). España

Producto de señalización vial horizontal CITICRYL D BLANCO - REF.:3129 es una pintura de señalización vial horizontal (tal como se define en la Norma EN 1871) que se emplea como material base para aplicarlo sobre la calzada sin necesidad de materiales agregados. La pintura se comercializa con las correspondientes instrucciones de aplicación. VALIDEZ: 30/12/2015.

\section{DITE 10-0444}

\section{DOS COMPONENTES F-3003 A/B}

\section{APLICACIÓN MÁQUINA 1:1}

FAPLISA (Fabricación y Aplicación de Pinturas Especiales S.A.)

c/ Paloma $n^{\circ}$ 13. Polígono Industrial Los Gallegos. 28946 FUENLABRADA (Madrid). España.

Producto de señalización vial horizontal DOS COMPONENTES F-3003 A/B APLICACIÓN MÁQUINA 1:1 es una pintura de señalización vial horizontal (tal como se define en la Norma EN 1871) que se emplea como material base para aplicarlo sobre la calzada requiriendo materiales agregados. La pintura (sistema plástico en frío) se comercializa con las correspondientes instrucciones de aplicación que incluyen los tipos y proporciones de materiales agregados.

VALIDEZ: 30/12/2015.

\section{DITE 10-0445}

PAISLANT ${ }^{\circledR}$ PANFRI, PANPLA,

\section{SANFRI, SANPLA}

AISLAMIENTOS PAIS S.L.

Polígono Industrial de Barros, Parcela 29/9-10.

39408 LOS CORRALES DE BUELNA (Cantabria). España

Sistema para cerramiento de cubiertas con paneles sándwich.

Los paneles PANFRI, PANPLA, SANFRI y SANPLA, objeto de DITE, consiste en paneles con cabios, de dos tipos:

Paneles tipo PAN-: fabricados con una capa interior o base, dos caras laterales de cabios de madera de pino, un núcleo de poliuretano inyectado y una barrera de papel para la protección del poliuretano contra la degradación por rayos UVA durante el proceso de instalación. Paneles tipo SAN-: paneles sándwich compuestos por una cara interior o base, dos caras laterales de cabios de pino, un núcleo de poliuretano inyectado y una cara externa de tablero de partículas hidrófugo. La unión entre el núcleo y las caras exteriores es llevada a cabo mediante inyección de poliuretano.

Los paneles están fijados con fijaciones mecánicas (puntas en L o tornillos) y juntas unidas por el exterior.

El sistema de paneles es usado como capa de cubierta aislante autoportante. Estos paneles son indicados para uso interior (siempre con recubrimiento exterior adicional), en condiciones de humedad normales. El sistema no tiene comportamiento estructural ni influye significativamente en la resistencia al arrastre en la obra.

Las previsiones indicadas en este DITE están basadas en una vida útil estimada de 25 años, para el sistema.

VALIDEZ: 30/12/2015.

\section{DITE 10-0446 \\ TEZNO T.6 CABIOS}

TEZNO CUBER COMPOSITES S.L.

c/ Majuelo $n^{\circ}$ 2. Polígono Cantabria 1-C. 26006 LOGROÑO (La Rioja). España.

Sistema para cerramiento de cubiertas con paneles sándwich.

Los paneles TEZNO T.6 CABIOS, objeto de DITE, consisten en paneles con cabios laterales de madera de pino. Las capas que lo componen son de naturaleza no metálica (friso de abeto o bien tablero de partículas hidrófugo + friso de abeto) y un núcleo de lana de roca de diferentes espesores.

Los paneles están fijados con fijaciones mecánicas (tornillos autotaladrantes o autorroscantes) y cuentan con junta machihembrada en su núcleo.

El sistema de paneles es usado como capa de cubierta aislante autoportante. Estos paneles son indicados para uso interior (siempre con recubrimiento exterior adicional), en condiciones de humedad normales. El sistema no tiene comportamiento estructural ni influye significativamente en la resistencia al arrastre en la obra.

Las previsiones indicadas en este DITE están basadas en una vida útil estimada de 25 años, para el sistema.

VALIDEZ: 30/12/2015.

\section{DITE 10-0447 \\ TEZNO T.1, TEZNO T.2, TEZNO T.3 Y TEZNO T.5}

TEZNO CUBER COMPOSITES S.L. c/ Majuelo $n^{\circ}$ 2. Polígono Cantabria 1-C. 26006 LOGROÑO (La Rioja). España. Sistema para cerramiento de cubiertas con paneles sándwich.
Los paneles TEZNO T. 1, T.2, T.3 y T.5 Pirineo, objeto de DITE, consisten en paneles sándwich.

Las capas que lo componen son de naturaleza no metálica (derivados de madera en su mayoría) y un núcleo de aislamiento térmico de poliestireno extruido de diferentes espesores unido mediante pegamento de poliuretano.

Los paneles están fijados con fijaciones mecánicas (tornillos autotaladrantes o autorroscantes) y cuentan con junta machihembrada en su núcleo.

El sistema de paneles es usado como capa de cubierta aislante autoportante. Estos paneles son indicados para uso interior (siempre con recubrimiento exterior adicional), en condiciones de humedad normales. El sistema no tiene comportamiento estructural ni influye significativamente en la resistencia al arrastre en la obra.

Las previsiones indicadas en este DITE están basadas en una vida útil estimada de 25 años, para el sistema.

VALIDEZ: 30/12/2015.

\section{DITE - 10/0448}

\section{TEZNO T. 4 GRANDES LUCES}

TEZNO CUBER COMPOSITES S.L. c/ Majuelo $n^{\circ}$ 2. Polígono Cantabria 1-C. 26006 LOGROÑO (La Rioja). España.

Sistema para cerramiento de cubiertas con paneles sándwich.

Los paneles TEZNO T. 1, T.2, T.3 y T.5 Pirineo, objeto de DITE, consisten en paneles sándwich.

Las capas que lo componen son de naturaleza no metálica (tableros de friso de abeto) y un núcleo de aislamiento térmico de poliestireno expandido de diferentes espesores unido mediante pegamento de poliuretano.

Los paneles están fijados con fijaciones mecánicas (tornillos autotaladrantes o autorroscantes) y cuentan con junta machihembrada en su núcleo.

El sistema de paneles es usado como capa de cubierta aislante autoportante. Estos paneles son indicados para uso interior (siempre con recubrimiento exterior adicional), en condiciones de humedad normales. El sistema no tiene comportamiento estructural ni influye significativamente en la resistencia al arrastre en la obra.

Las previsiones indicadas en este DITE están basadas en una vida útil estimada de 25 años, para el sistema.

VALIDEZ: 30/12/2015. 